Review Paper

\title{
Optimization and selection of the multi-objective conceptual design scheme for considering product assembly, manufacturing and cost
}

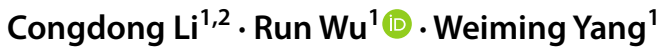 \\ Received: 9 September 2021 / Accepted: 3 February 2022 \\ Published online: 04 March 2022 \\ (c) The Author(s) 2022 OPEN
}

\begin{abstract}
Conceptual design is the crucial stage of selecting and determining product composition and configuration, which greatly affects product performance and cost. In conceptual design stage, designers have the maximum design freedom in order to put forward to the optimal design solution in terms of assembly, manufacturing and cost. However, the above product aspects are analyzed independently in current industry, and a guided decision-making method based on multi-objective criteria is missing. Therefore, it is necessary to introduce a multi-objective analysis method to obtain an optimal conceptual scheme considering various aspects of product. Due to the complexity of product conceptual solution generation, this study divides the multi-objective analysis process into multi-objective solution optimization and multi-objective solution selection. The non-dominated solution set can be obtained from all the potential solutions in the former step. Then, the optimal conceptual solution can be obtained from the non-dominated solutions set in the latter step. The two-stage multi-objective analysis method has two advantages: (1) large dominated solutions can be quickly eliminated which can reduce the workload of solution selection; (2) TOPSIS method is easy to implement, which can work with attribute weights and scores of each design option. Finally, using the conceptual design of a centrifugal compressor as an example, the method's availability and practicality are demonstrated.
\end{abstract}

Keywords Conceptual design $\cdot$ Design for X $\cdot$ Multi-objective optimization $\cdot$ Multi-objective selection

\section{Introduction}

Design-for- $X(\mathrm{DfX})$ methods are used to aid designers during the design process from a specific perspective $X$ [1]. As the perspective of assembly of DfX, DfA supports designers to optimize components assembly. At present, the main methods of DfA include Boothroyd and Dewhurst (B\&D) method and product function decomposition method [2]. The previous method uses the quantitative indicator to assess assembly complexity. However, this method is quite time-consuming since it relies largely on existing product prototypes. The latter method assesses product assembly complexity through the overall product functional decomposition, which can be applied in product conceptual design phase [3, 4]. However, the conceptual DfA method aims at the product assembly activities, other product aspects such as manufacturability and cost not considered. Furthermore, product conceptual design and optimization is a multi-objective activity that is not just concerned with components assembly.

In this regard, the given article provides a solution to the aforementioned inadequacies and optimizes product conceptual design by taking into account various aspects of product, such as manufacturing and cost. When the product prototype is infeasible, the proposed method can be used to optimize the comprehensive aspects of product

Run Wu, 597028824@qq.com | ${ }^{1}$ School of Management, Jinan University, Guangzhou 510632, Guangdong, China. ${ }^{2}$ Institute of Physical Internet, Jinan University, Guangzhou 510632, Guangdong, China.

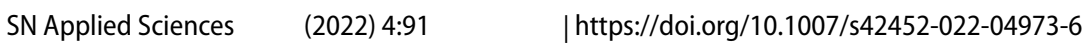


in conceptual design phase. The main motivation of the proposed multi-objective method is to support designers with the trade-off analysis among product attributes, which is crucial to comprehensively optimize the product conceptual process. The innovative aspect of this method consists of comprehensive considering various product aspects in conceptual design phase. Moreover, a mathematical model based on the multi-criteria decisionmaking (MCDM) theory makes this method feasible and applicable.

The remainder of this work is structured as follows: Sect. 2 provides an overview of the research history in this subject. The suggested multi-objective design method is explained in detail in Sect. 3. Section 4 transforms the challenge of multi-objective conceptual scheme creation into a multi-objective optimization problem that can be handled using multiple objective particle swarm optimization (MOPSO). Section 5 examines the conceptual design of a centrifugal compressor as a case study. Section 6 has a discussion and concluding remarks.

\section{Literature review}

The product design process can be divided into four stages: (1) design task definition, (2) conceptual design, (3) embodiment design, and (4) detailed design [5]. In the first phase, the overall design task can be definited by the customer requirements collected. Then, the design task is translated into functions and features in a specific process model. Finally, the above conceptual model is implemented step by step. As the crucial stage in product design process, conceptual design determines the most performance and costs of product.

DfA is a systematic analysis process to aid and guide designers to assemble in an efficient manner [6]. The primary motivation of DfA analysis is to maximize use of the same components and reduce the assembly cost and time [2]. Boothroyd and Dewhurst proposed the Boothroyd-Dewhurst (B\&D) method for DFA in 1980, which is one of the most widely applied methods in the industry and business by some companies, such as G.E and IBM Crop. The B\&D method can be used to calculate the product design's assembly efficiency for both manual and automatic operation. When implemented at conceptual design phase, DfA method may strongly enhance the product assembly performance and reduce the cost in terms of components assembly. However, the traditional DfA method is usually applied in the detailed phase when the main design process has been determined, which is the main disadvantage of these methods $[3,4]$.

In order to make up for the deficiency of B\&D method, Stone et al. [3] proposed a conceptual DfA method to support conceptual design activities. Two basic concepts are defined in this method: functional basis and the module heuristics method. Functional basis is utilized to generate a functional model by function decomposition, and the module heuristic method is used to identify a modular product architecture [6]. In Pahl and Beitz theory, a function-based conceptual design process model is proposed, and functional basis can be obtained by function definition and its decomposition [7]. In this way, the overall function can be converted into several sub-functions, which is relatively easier to be achieved by the modules heuristics method. However, the conceptual DfA still has two week points: (1) only the product assembly process is considered; and (2) the process of the optimal solution generation is not discussed in detail, such as the optimization process and selection process.

Besides DfA, methods of DfX include the consideration of several issues such as design for manufacturing (DfM) and design for cost (DfC) [8-12]. DfM is defined as a method for determining the product manufacturing aspects where (i) manufacturing cost; (ii) transform design into production; (iii) the manufactured product are required to meet product quality requirements. It can be seen that DfM affects the product performance and cost through the design and manufacturing transformation process.

Product cost estimation (PCE) research covers a wide variety of topics, ranging from manufacturing components cost estimation to cost analysis of highly customized assembled products, from process cost estimation method to the specific methods of overall product cost calculating [13-16]. Among them, design-to-cost (DtC) method can be broadly classified into three types: (1) intuitive cost estimation, (2) parametric estimation, and (3) analogous estimation. In the detailed design phase, the most accurate cost estimation method is intuitive methods. Among these methods, the most commonly used method is based on knowledge, features and the similarity of previous data. To obtain and appropriate estimation method, DtC must be applied at the same time as DfM and DfA applied so as to make the design alternative solutions more cost-efficient.

In order to overcome the above issues, a multi-objective method that considers various product aspects (assembly, manufacturing, cost, etc.) in conceptual design is proposed. The process of multi-objective conceptual design can be summered as two sub stages: (1) multi-objective optimization of solutions, and (2) the optimal solution selection. The process of multi-objective solutions optimization is completed by multi-objective particle swarm optimization (MOPSO) [17], and the optimal solution selection is completed by a MCDM (multi-criteria decisionmaking) method called TOPSIS [18]. Among many algorithms for multi-objective optimization problem, MOPSO 
algorithm has unique advantages. Firstly, compared with genetic algorithm (GA), the implementation of particle swarm optimization algorithm is simpler and easier. Secondly, PSO algorithm is a population-based evolutionary algorithm. Each iteration of PSO can generate a set of nondominant solutions. After appropriate expansion, it can be used to solve multi-objective optimization problems. TOPSIS method obtains the closest solution to the ideal solution by sort each solution distance to the positive ideal solution and negative ideal solution. The advantages of TOPSIS include: (1) its utility function is monotonic; (2) its solving process is simple and easy to understand; (3) convert the scheme parameters that are not easy to calculate into simple mathematical calculation; (4) it can be applied to different scheme selection problems.

\section{Basic framework}

This section discusses the basic concepts and framework of the multi-objective conceptual design method. To begin, several fundamental concepts must be introduced. The theory of product modules and features is developed considering the functional basis and module heuristics. Feasible design solutions are defined and analyzed as the morphological matrix. The general expression of multiobjective problem is presented. Second, the objectives and composition of conceptual design scheme are introduced. Finally, the process of multi-objective conceptual design scheme optimization and selection is presented. These concepts and framework are further discussed in the sub-sections in detail.

\subsection{Product module and property definition}

The first step in this method is to define product modules and characterize module features. The sub-functions and modules can be determined through the relative functional analysis and module heuristic method. As the first step of product conceptual design, functional analysis allows the product overall function to be broken down a series of sub-functions. Functional analysis can help design experts determine the functional structure of product.

The process of functional modeling refers to express the overall function and sub-functions with the input/out flows (material, energy and signal). The method of module heuristics can help design experts identify product modules from the analysis of input/output flows of these sub-function. After the conversion, the product overall function can be translated into modules. The mapping between sub-functions and modules is complex. In order to highlight the research focus in this article, the mapping is simplified as one-to-one mapping.
Furthermore, some specific functional module properties need to be determined in heuristics method. Module properties refer to the product aspects that need to be determined during product conceptual design. There are three typical module properties considered in this article: assembly, manufacturing and cost. These module properties can be described quantitatively by corresponding attributes and scores. The definition of module and property is the basis for definition of feasible and unfeasible design solutions in terms of both benefit attributes and cost attributes in production.

\subsection{Identification of feasible design solutions}

The transformation from product modules to potential design solutions is based on the specific attributes identified during the generation of product modules. A very helpful tool during this step is the morphological matrix, which can improve the effectiveness of conceptual analysis. A morphological matrix is traditionally created by labeling rows with all identified product modules and columns with the possible options. The morphological matrix shows existing alternative design options for each functional module and permits rapid configuration of the product with selection the best option for specific module. Design options must be reliable and compliant with the attributes defined in the module assessment. Shown as Table 1, there are four functional modules and each functional module has four options. If we specify that only one option can be selected for each module, a total of 16 feasible solutions can be obtained.

In order to optimize and select the optimal solution from these feasible solutions, it is necessary to score according to the following three module properties: assembly, manufacturing and cost. These scores are given by the corresponding design experts. The scores represent the module performance of the selected option in the corresponding property (Tables 2, 3, 4).

\subsection{Conceptual design scheme}

In this article, the product conceptual scheme consists of sub-functions and modules. The process of product

Table 1 Feasible design solutions generated in morphological matrix

\begin{tabular}{lllll}
\hline & Option 1 & Option 2 & Option 3 & Option 4 \\
\hline Functional module 1 & A1 & A2 & A3 & A4 \\
Functional module 2 & B1 & B2 & B3 & B4 \\
Functional module 3 & C1 & C2 & C3 & C4 \\
Functional module 4 & D1 & D2 & D3 & D4 \\
\hline
\end{tabular}


Table 2 The module-option scores in assembly property

\begin{tabular}{lllll}
\hline & Option 1 & Option 2 & Option 3 & Option 4 \\
\hline Functional module 1 & $\mathrm{a} 1 \mathrm{a}$ & $\mathrm{a} 2 \mathrm{a}$ & $\mathrm{a} 3 \mathrm{a}$ & $\mathrm{a} 4 \mathrm{a}$ \\
Functional module 2 & $\mathrm{b} 1 \mathrm{a}$ & $\mathrm{b} 2 \mathrm{a}$ & $\mathrm{b} 3 \mathrm{a}$ & $\mathrm{b} 4 \mathrm{a}$ \\
Functional module 3 & $\mathrm{c1a}$ & $\mathrm{c} 2 \mathrm{a}$ & $\mathrm{c} 3 \mathrm{a}$ & $\mathrm{c4a}$ \\
Functional module 4 & $\mathrm{d} 1 \mathrm{a}$ & $\mathrm{d} 2 \mathrm{a}$ & $\mathrm{d} 3 \mathrm{a}$ & $\mathrm{d} 4 \mathrm{a}$ \\
\hline
\end{tabular}

Table 3 The module-option scores in manufacturing property

\begin{tabular}{lllll}
\hline & Option 1 & Option 2 & Option 3 & Option 4 \\
\hline Functional module 1 & $\mathrm{a} 1 \mathrm{~m}$ & $\mathrm{a} 2 \mathrm{~m}$ & $\mathrm{a} 3 \mathrm{~m}$ & $\mathrm{a} 4 \mathrm{~m}$ \\
Functional module 2 & $\mathrm{b} 1 \mathrm{~m}$ & $\mathrm{~b} 2 \mathrm{~m}$ & $\mathrm{~b} 3 \mathrm{~m}$ & $\mathrm{~b} 4 \mathrm{~m}$ \\
Functional module 3 & $\mathrm{c} 1 \mathrm{~m}$ & $\mathrm{c} 2 \mathrm{~m}$ & $\mathrm{c} 3 \mathrm{~m}$ & $\mathrm{c} 4 \mathrm{~m}$ \\
Functional module 4 & $\mathrm{d} 1 \mathrm{~m}$ & $\mathrm{~d} 2 \mathrm{~m}$ & $\mathrm{~d} 3 \mathrm{~m}$ & $\mathrm{~d} 4 \mathrm{~m}$ \\
\hline
\end{tabular}

Table 4 The module-option scores in cost property

\begin{tabular}{lllll}
\hline & Option 1 & Option 2 & Option 3 & Option 4 \\
\hline Functional module 1 & $\mathrm{a} 1 \mathrm{c}$ & $\mathrm{a} 2 \mathrm{c}$ & $\mathrm{a} 3 \mathrm{c}$ & $\mathrm{a} 4 \mathrm{c}$ \\
Functional module 2 & $\mathrm{b} 1 \mathrm{c}$ & $\mathrm{b} 2 \mathrm{c}$ & $\mathrm{b} 3 \mathrm{c}$ & $\mathrm{b} 4 \mathrm{c}$ \\
Functional module 3 & $\mathrm{c} 1 \mathrm{c}$ & $\mathrm{c} 2 \mathrm{c}$ & $\mathrm{c} 3 \mathrm{c}$ & $\mathrm{c} 4 \mathrm{c}$ \\
Functional module 4 & $\mathrm{~d} 1 \mathrm{c}$ & $\mathrm{d} 2 \mathrm{c}$ & $\mathrm{d} 3 \mathrm{c}$ & $\mathrm{d} 4 \mathrm{c}$ \\
\hline
\end{tabular}

$\operatorname{Max} \quad F(x)=\left[f_{1}(x), f_{2}(x), \ldots, f_{l}(x)\right]$

Find $x=\left[x_{1}, x_{2}, \ldots, f_{l}(x)\right]$

$g_{i}(x)<0, i=1,2, \ldots, m$

$h j(x)=0, j=1,2, \ldots, n$

where $f_{i}(x)(i=1,2, \ldots, l)$ is the objective function. $X=\left[x_{1}\right.$, $\left.x_{2}, \ldots, x_{t}\right]$ is the design variable, and $t, l, m, n$ are the number of design variables, objective functions, equality constraints and inequality constrains.

In multi-objective optimization, each objective restricts each other through design variables. The optimization of one objective must be at the expense of other objectives, so it is difficult to find the real optimal solution. The essential difference between multi-objective optimization and single objective optimization is that the solution of multiobjective optimization is not unique, but there are a series of solutions. Its characteristic is that at least one objective is better than all other solutions. Such solutions are called non-dominated solutions or optimal solutions, and these solutions are Pareto optimal solution sets.

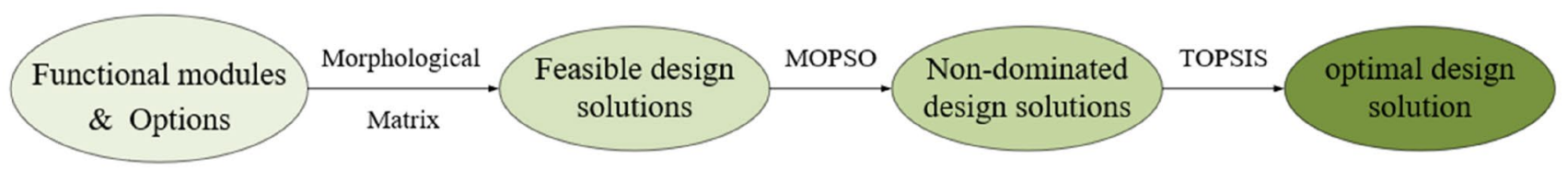

Fig. 1 The generation process of the optimal solution

conceptual scheme generation can be summarized as four steps: (1) decomposition the overall product function into several sub-functions; (2) translate these sub-functions into functional modules and generate potential solutions by the morphological matrix; (3) optimize these solutions to obtain the non-dominated solution set by MOPSO; (4) select the optimal solutions from above solution set by TOPSIS.

In order to facilitate understanding and processing, the design solutions are divided into three types: feasible design solutions, non-dominated solutions and the optimal solution. Figure 1 shows the relationship between the three type solutions.

\subsection{Multi-objective optimization}

The multi-objective optimization problem can be expressed as:

\section{Multi-objective design approach}

The solution of multi-objective problem is not only an optimization problem. After the Pareto optimal solution set is obtained, the final compromise solution or optimal solution needs to be selected according to the decision theory. In this proposed approach, the optimization method of the conceptual scheme is multi-objective particle swarm optimization (MOPSO). The selection of the best design scheme is made using a MCDM method called TOPSIS. Using these method, the different design options, identified in the previous step, are ranked so as to choose the best module configuration taking into account several aspects such as assemblability, manufacturability, materials and costs. The TOPSIS method is not time-consuming due to easy implementation in a common spreadsheet or in dedicated software. Inputs required are only (1) 
attribute weights (based on designers and information entropy) and (2) values for each design option in relation to the selected attributes.

\subsection{Particle swarm optimization (PSO)}

Particle swarm optimization is a new intelligent algorithm after GA, SA and ACO. PSO solves the optimal problem through the coordination of the optimal direction of the group, the optimal direction of the individual and the inertial direction by imitating the mechanism of coordination between the individual and the whole in the overall foraging and migration of birds.

Let the population size of particles be $\mathrm{m}$ and the decision space be $n$-dimensional, where the coordinate position of particle $i$ at time $t$ can be expressed as $X_{i}^{t}=\left(x_{i 1}^{t}, x_{i 2}^{t}, \ldots, x_{i m}^{t}\right)$. The distance that particle $i$ moves in each iteration is defined as the particle velocity $V_{i}^{t}=\left(v_{i 1}^{t}, v_{i 2}^{t}, \ldots, v_{i m}^{t}\right)$. Then, the flight speed and position of particle $i$ in the $j(i=1,2, \ldots, n)$ dimensional subspace of time $t$ can be adjusted according to the following formulas.

$v_{i j}^{t}=w v_{i j}^{t-1}+c_{1} r_{1}\left(p_{\text {best }}-x_{i j}^{t-1}\right)+c_{2} r_{2}\left(g_{\text {best }}-x_{i j}^{t-1}\right) ;$

$x_{i j}^{t}=x_{i j}^{t-1}+v_{i j}^{t} ;$

In above formulas, $r_{1}$ and $r_{2}$ are two random numbers in the range of $[0,1]$. The inertia weight of the particle is denoted as $w$, acceleration factors as $c_{1}$ and $c_{2}$, the individual extreme value as $P_{\text {best }}$ and the global extremum as $G_{\text {best }}$. In addition to considering the Pareto dominance of particles, we need to consider how to maintain the diversity of particle swarm to avoid converging to a single solution. In this article, maxminPSO method proposed in reference is used to obtain Pareto sets. MaxminPSO uses fitness function to determine Pareto dominating solution in multi-objective optimization process. The test example shows that maxminPSO can obtain a more real and uniform Pareto front than NSGA-II. The solution flow is shown in Fig. 2.

\subsection{TOPSIS method based on combination weighting}

Since the weight of index evaluation is involved in the process of scheme evaluation, this article introduces combined weight to obtain the weight of each index. The expert opinion is regarded as the subjective weight, and the entropy weight method is selected for the objective weight. In information theory, information is a measure of the degree of order of the system. Information entropy measures the importance of the attribute in decision-making, that is, if the smaller the information entropy of an index, the greater the amount of information provided by the index. The higher the importance in comprehensive evaluation, the weight should be greater. The TOPSIS solution process based on combination weighting can be divided into the following six steps.

Step 1: Establishing standard decision matrix.

For the multi-attribute problem with $\mathrm{m}$ alternatives and $N$ attributes, the initial decision matrix is shown as follow.

$A=\left(\begin{array}{ccc}y_{11} & \cdots & y_{1 n} \\ \vdots & \ddots & \vdots \\ y_{m 1} & \cdots & y_{m n}\end{array}\right)$

Normalize the initial matrix $A$ into a matrix.

$B=\left(\begin{array}{ccc}b_{11} & \cdots & b_{1 n} \\ \vdots & \ddots & \vdots \\ b_{m 1} & \cdots & b_{m n}\end{array}\right)$

where $b_{i j}$ is the standard value of the $i$-th alternative on the $j$-th evaluation index. The benefit formula is shown as follow.

$b_{i j}=\frac{y_{i j}-y_{\min }}{y_{\mathrm{ma}} x-y_{\min }} ;$

The cost formula is shown as follow.

$b_{i j}=\frac{y_{\max }-y_{i j}}{y_{\operatorname{ma}} x-y_{\min }} ;$

Step 2: Calculating entropy weight.

$e_{j}$ was defined as the entropy of $j$-th indicators.

$e_{j}=-k \sum_{i=1}^{n} p_{i j} \ln p_{i j}$.

$p i j=\frac{b_{i j}}{\sum_{i=1}^{n} b_{i j}} ; k=\frac{1}{\ln (n)}$

Since the smaller the information entropy, the greater the weight. The weight of $j$-th indicators is as follow:

$w_{j}=\frac{1-e_{j}}{\sum_{j=1}^{n}\left(1-e_{j}\right)} ;$

where $w_{j} \in[0,1] ; \sum_{j=1}^{n} w_{j}=1, j=1,2, \ldots, n$.

Step 3: The entropy weight is modified by expert weighting to obtain the combined weight, and the canonical weighting matrix $C$ is constructed based on the combined weight. The weight to $j$-th indicators is 
Fig. 2 The main process of MOPSO method

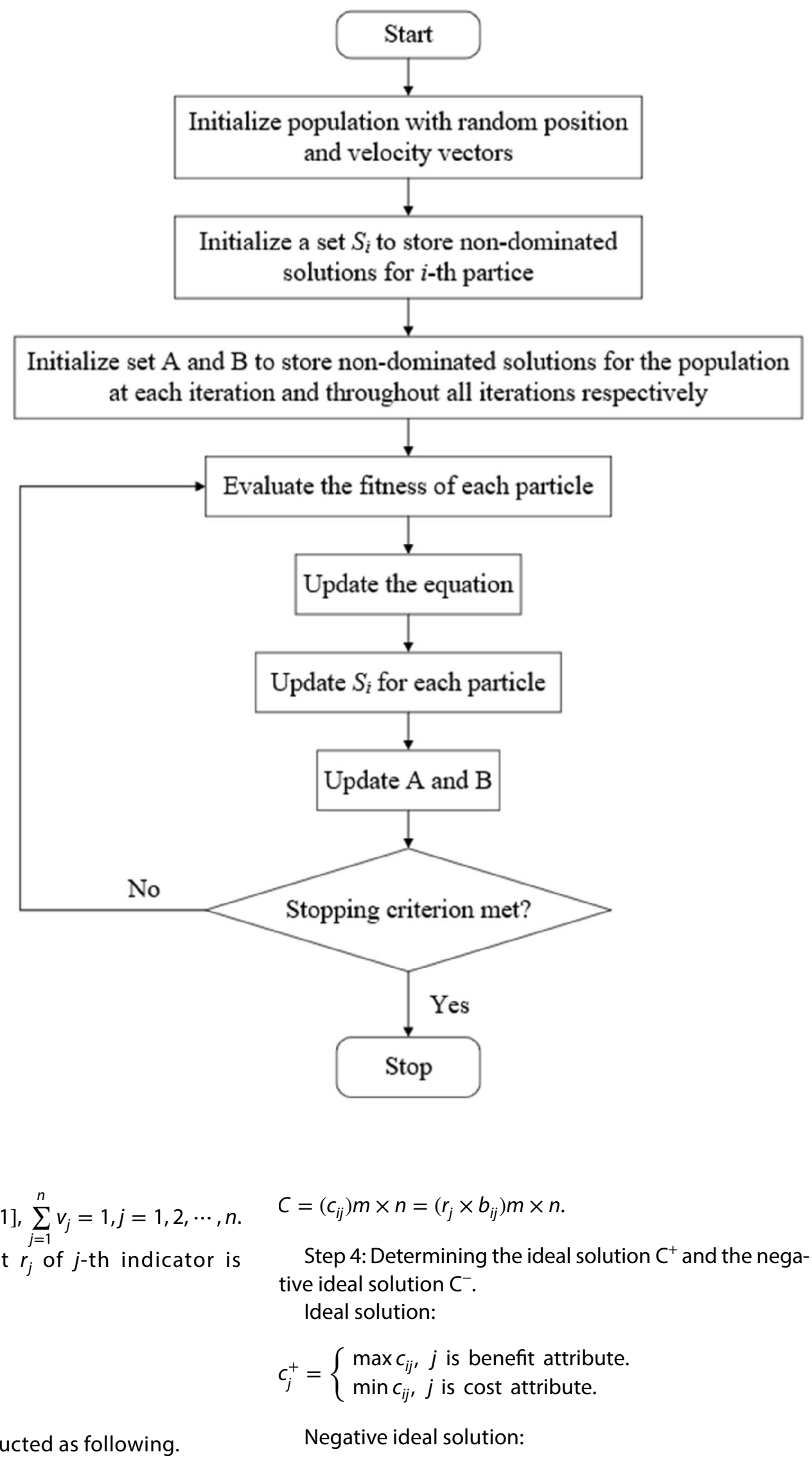
solutions for $i$-th partice denoted as $v_{j}$, where $v_{j} \in[0,1], \sum_{j=1}^{n} v_{j}=1, j=1,2, \cdots, n$. Then, the combined weight $r_{j}$ of $j$-th indicator is determined.

$r_{j}=\frac{v_{j} w_{j}}{\sum_{j=1}^{n} v_{j} w_{j}}, j=1,2, \cdots, n$

Normalized matrix is constructed as following. 
$c_{j}^{-}=\left\{\begin{array}{l}\min c_{i j}, j \text { is benefit attribute. } \\ \max c_{i j} j \text { is cost attribute. }\end{array}\right.$

Step 5: Calculating the Euclidean distance from each alternative to the ideal solution and the negative ideal solution $d_{i}^{+}$and $d_{i}^{-}$.

$d_{i}^{+}=\sqrt{\sum_{j=1}^{n}\left(c_{i j}-c_{j}^{+}\right)^{2}}, i=1,2, \ldots, m ;$

$d_{i}^{-}=\sqrt{\sum_{j=1}^{n}\left(c_{i j}-c_{j}^{-}\right)^{2}}, i=1,2, \ldots, m ;$

Step 6: Calculating the evaluation index $p_{i}$ of each solution.

These solutions can be arranged from large to small according to $p_{i}$. The relative distance is used to reflect the degree to which the solution is close to the ideal solution and the negative ideal solution as shown in the following formula. The larger the $p_{i}$, the better the solution.

$p_{i}=d_{i}^{-} /\left(d_{i}^{+}+d_{i}^{-}\right), i=1,2, \ldots, m$.

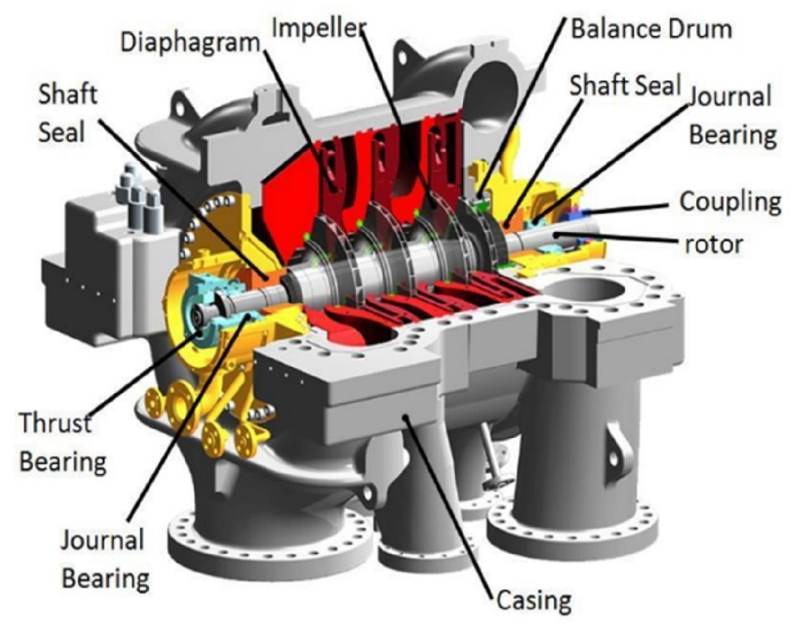

Fig. 3 The main components of the centrifugal compressor

\section{Case study}

The centrifugal compressor is a type of turbo-compressor that raises gas pressure by adding kinetic energy/velocity to a continuous flow of fluid through impellers. By slowing the flow via a diffuser, the kinetic energy is transformed to a rise in pressure. The centrifugal compressor's major components are seen in Fig. 3: impeller, balancing drum, shaft seal, diaphragm, coupling, rotor, casing, journal bearing and thrust bearing. The generating process of the centrifugal compressor conceptual design plan validates the feasibility and efficacy of the proposed method.

\subsection{Functional basis and modules of the centrifugal compressor}

Constructing the function model is the initial stage in the multi-objective design method. The overall design requirements may be broken down into many reasonably separate functional basis based on the centrifugal compressor's major components. Figure 3 depicts the functional structure diagram of centrifugal compressors, in which the functional basis is implemented using matching modules. The centrifugal compressor's nine components are examined in this study. Impeller is one of them, and its too complicated to be broken down into five basic functioning parts any more. Because the basic solution for the balancing drum is straightforward, it will not be examined. As a result, the following 10 functional basis components are proposed: $\mathrm{FB}_{1}$ (impeller blade bending form), $\mathrm{FB}_{2}$ (impeller blade type), $\mathrm{FB}_{3}$ (impeller blade shape), $\mathrm{FB}_{4}$ (impeller structure), $\mathrm{FB}_{5}$ (impeller construct technology), $\mathrm{FB}_{6}$ (coupling), $\mathrm{FB}_{7}$ (casing), $\mathrm{FB}_{8}$ (diaphragm), $\mathrm{FB}_{9}$ (shaft seal) and $\mathrm{FB}_{10}$ (bearings).

The suggested method's second step is to look for the appropriate modules of these functional basis. Using the morphological matrix, all the potential design solutions can be investigated (e.g., part of design potential solution as Table 5). The relevant modules may be discovered as illustrated in Fig. 4 under the restrictions of current resources. The morphological matrix can assist designers in swiftly generating creative design solutions. However, there is a challenge to this method: the vast number of
Table 5 Example of morphological matrix for part modules

\begin{tabular}{llll}
\hline Module & Option & & \\
\cline { 2 - 3 } & Design option 1 & Design option 2 & Design option 3 \\
\hline Impeller blade bending form & Back & Radial & Forward \\
Impeller structure & Open & Semi-enclosed & Enclosed \\
Casing & Horizontal section & Vertical section & Volute section \\
$\ldots \ldots$. & $\ldots \ldots$ & $\ldots \ldots$ & $\ldots .$. \\
\hline
\end{tabular}




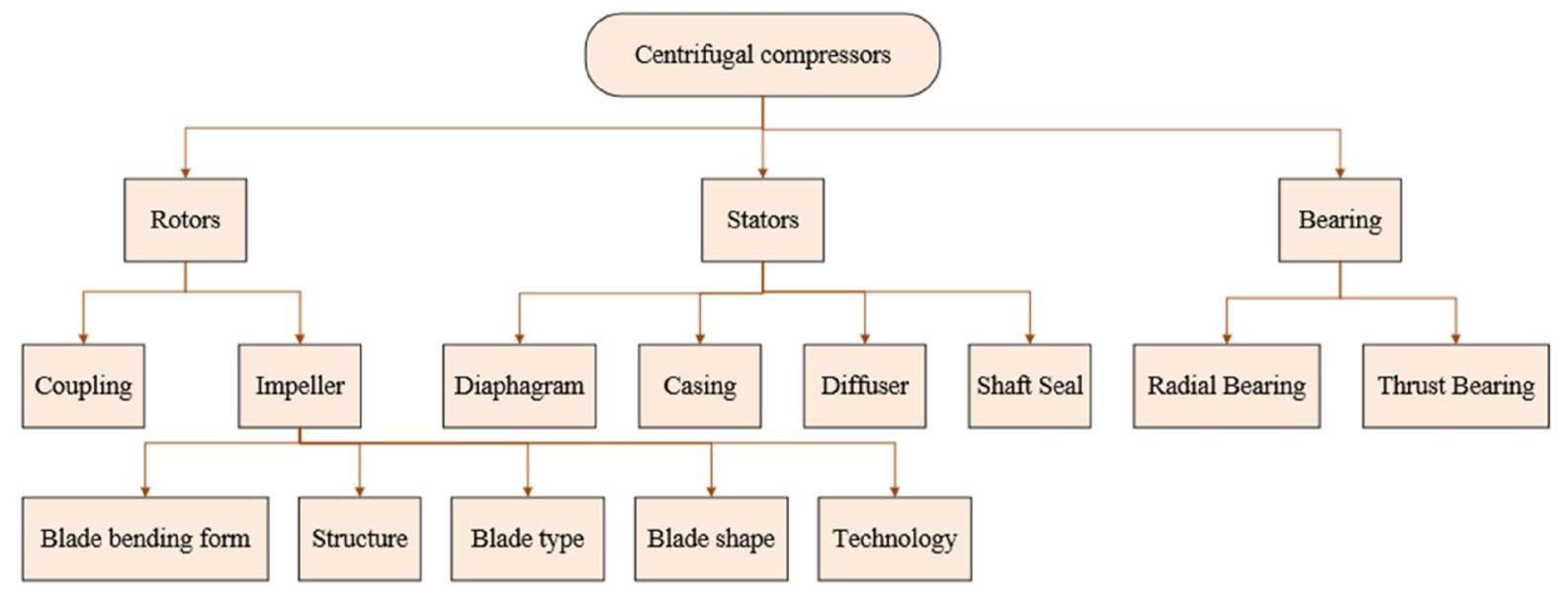

Fig. 4 The functional structure of the centrifugal compressor

alternatives makes the solutions space too large to select the best one. The next step is to optimize and select these potential solutions (Fig. 5).

\subsection{Optimization of the potential design solutions}

To facilitate modeling, following decision variables are defined.
Definition 1: $x_{i j}(i=1,2, \ldots, m ; j=1,2, \ldots, n)$ is a $0-1$ variable. If the $i$-th functional basis and $j$-th modules is selected, $x_{i j}=1$; otherwise, $x_{i j}=0$.

Definition 2: $y_{i j}\left(i=1,2, \ldots, m_{i j}=1,2, \ldots, n\right)$ is a numerical variable, and $y_{i j}$ is the assembly attribute value of the $i$-th functional basis and $j$-th module.

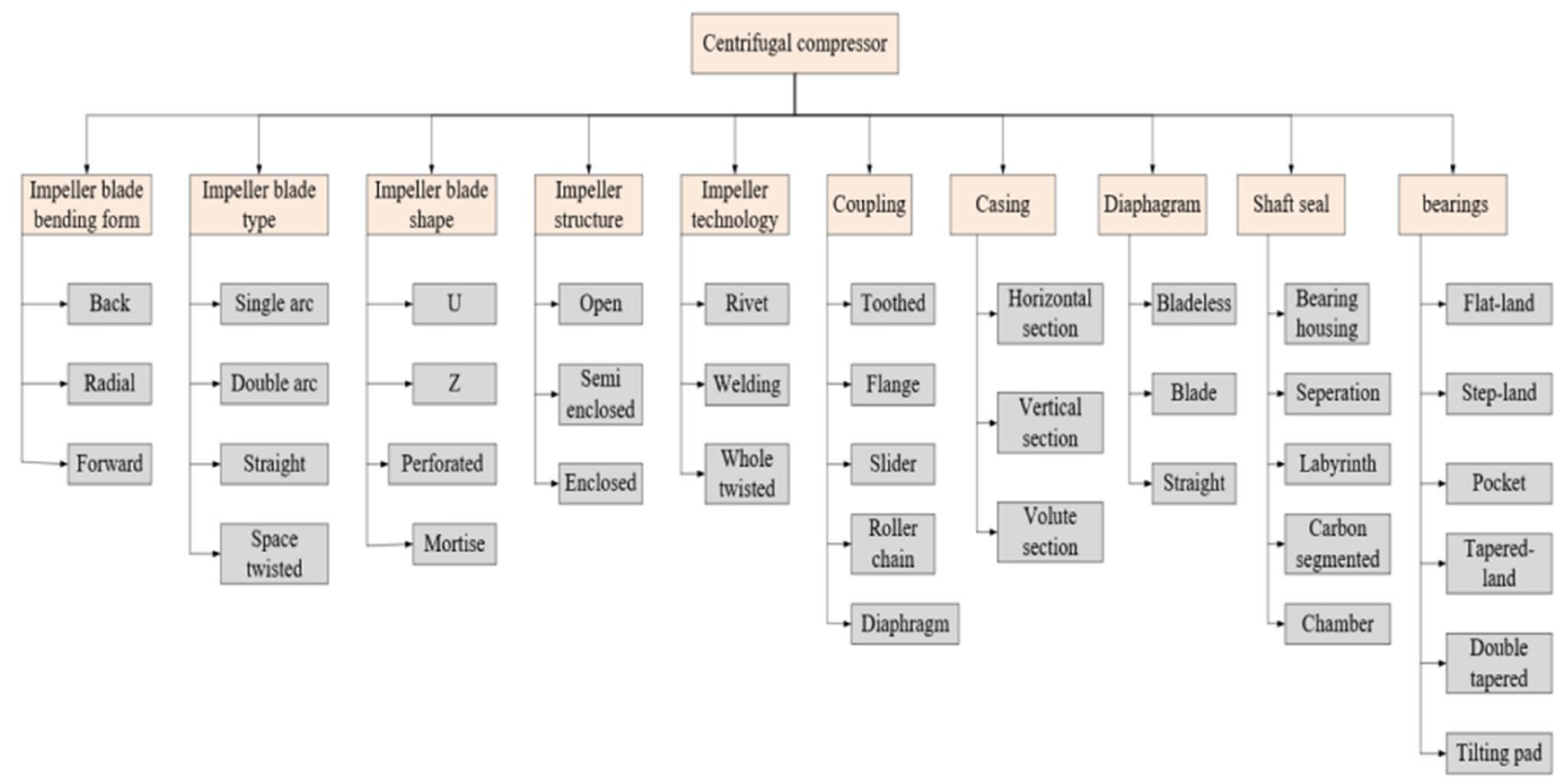

Fig. 5 The functional basis and modules of centrifugal compressor 
Definition 3: $z_{i j}(i=1,2, \ldots, m ; j=1,2, \ldots, n)$ is a numerical variable, and $z_{i j}$ is the manufacturing attribute value of the $i$-th functional basis and $j$-th module.

Definition 4: $k_{i j}\left(i=1,2, \ldots, m_{i j}=1,2, \ldots, n\right)$ is a numerical variable, and $k_{i j}$ is the cost attribute value of the $i$-th functional basis and $j$-th module.

By taking the objective function maximization of assembly attributes $F(A)$, maximization of manufacturing attributes $F(M)$ and the objective function minimization of cost attribute $F(C)$, the multi-objective optimization model is as follows:

$F(x)=[\max F(A), \max F(M), \min F(C)]$

$F(A)=\sum_{i=1}^{m} \sum_{j=1}^{n} x_{i j} y_{i j}$

$F(M)=\sum_{i=1}^{m} \sum_{j=1}^{n} x_{i j} z_{i j}$

$F(C)=\sum_{i=1}^{m} \sum_{j=1}^{n} x_{i j} k_{i j}$

The relevant constraints conditions are as follows: $\sum_{j=1}^{n} x_{i j}=1$

$D_{l}^{*} \leq D_{i}$

According to above analysis of centrifugal compressor, the design variable is $x_{i j}, y_{i j}, z_{i j}$ and $k_{i j}$. The value of $y_{i j}$ is determined by design experts' score on the assembly attribute of the $j$-th module. Similarity, the value of $z_{i j}$ and $k_{i j}$ is determined by design experts' score on the manufacturing and cost attribute of the $j$-th module. The scale of the assembly attribute, manufacturing attribute and cost attribute value is from 1 to 10 .

Therefore, the value of $y_{i j}$ and $z_{i j}$ of can be obtained as Tables 6, 7 and 8 .

The objectives of the optimization problem is to maximize the $F(A), F(M)$ and minimizing $F(C)$. In the multi-objective optimization model, we considered the population and repository size is 200 , and maximum generation is 2000 . To solve the multi-objective optimization problem and obtain the Pareto frontier, the MOPSO algorithm is coded and run in MATLAB R2016b software environment. The dominant schemes are calculated among all candidates, and the results are shown in Fig. 6. After calculation, there are 69 non-dominant solutions in Pareto frontier (Table 9).

Table 6 The module-option scores in assembly property

\begin{tabular}{lllllllllll}
\hline & Module1 & Module2 & Module3 & Module4 & Module5 & Module6 & Module7 & Module8 & Module9 & Module10 \\
\hline Option 1 & 7 & 6 & 3 & 8 & 8 & 7 & 3 & 6 & 6 & 3 \\
Option 2 & 8 & 3 & 8 & 4 & 5 & 3 & 6 & 7 & 5 & 4 \\
Option 3 & 3 & 4 & 5 & 2 & 3 & 4 & 5 & 3 & 3 & 7 \\
Option 4 & 0 & 8 & 4 & 0 & 0 & 4 & 0 & 0 & 2 & 5 \\
Option 5 & 0 & 0 & 0 & 0 & 0 & 7 & 0 & 0 & 6 & 6 \\
Option 6 & 0 & 0 & 0 & 0 & 0 & 0 & 0 & 0 & 0 & 8 \\
\hline
\end{tabular}

Table 7 The module-option scores in manufacturing property

\begin{tabular}{lllllllllll}
\hline & Module1 & Module2 & Module3 & Module4 & Module5 & Module6 & Module7 & Module8 & Module9 & Module10 \\
\hline Option 1 & 5 & 2 & 6 & 8 & 8 & 3 & 3 & 6 & 8 & 3 \\
Option 2 & 7 & 6 & 4 & 4 & 5 & 4 & 6 & 7 & 6 & 7 \\
Option 3 & 3 & 7 & 6 & 7 & 3 & 3 & 6 & 3 & 3 & 5 \\
Option 4 & 0 & 3 & 4 & 0 & 0 & 6 & 0 & 0 & 2 & 5 \\
Option 5 & 0 & 0 & 0 & 0 & 0 & 5 & 0 & 0 & 4 & 3 \\
Option 6 & 0 & 0 & 0 & 0 & 0 & 0 & 0 & 0 & 0 & 7 \\
\hline
\end{tabular}


Table 8 The module-option scores in cost property

\begin{tabular}{lllllllllll}
\hline & Module1 & Module2 & Module3 & Module4 & Module5 & Module6 & Module7 & Module8 & Module9 & Module10 \\
\hline Option 1 & 3 & 5 & 1 & 6 & 6 & 10 & 5 & 3 & 10 & 7 \\
Option 2 & 4 & 3 & 7 & 2 & 3 & 1 & 3 & 5 & 10 & 3 \\
Option 3 & 2 & 3 & 2 & 4 & 2 & 5 & 2 & 2 & 6 & 10 \\
Option 4 & - & 7 & 4 & - & - & 3 & - & - & 1 & 10 \\
Option 5 & - & - & - & - & - & 5 & - & - & 3 \\
Option 6 & - & - & - & - & - & - & - & - & - & 6 \\
\hline
\end{tabular}

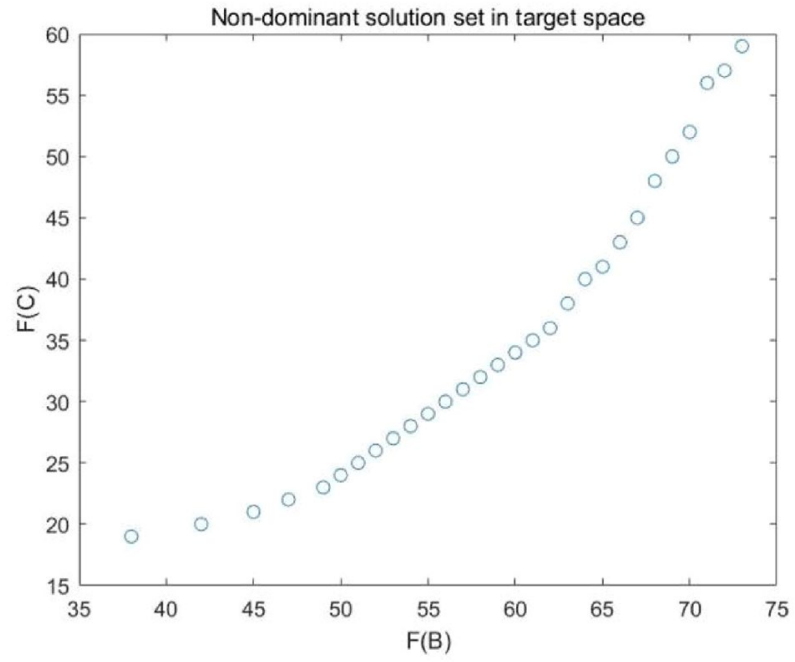

Fig. 6 The non-dominant solution set of centrifugal compressor

\subsection{Selection of the optimal design solution from the non-dominated solution sets}

Based on designer experience, the weight vector $v=(0.5$, $0.15,0.15)$ of the three design objectives is obtained. The objective weight $w=(0.07,0.24,0.69)$ can be obtained by the information entropy weight method. The combined weight vector $=(0.11,0.13,0.77)$ is obtained by the formula. The optimal solution can be selected from the Pareto solution sets with the expert weighting method, information entropy method and the combined method.

It can be seen from Table 10 that the optimal solution $A$ obtained buy expert weighting method which focus on the assembly attributes. The optimal solution $B$ is obtained by the information entropy weighting method which lack of authenticity. The combined weighting method is applied to obtain the optimal solution $C$, which compromises between expert weighting method and information entropy weight method. The module options selected are listed in the following table.

\section{Discussion}

The presented case study (centrifugal compressor) is a good example for demonstrating the advantages of a multi-objective approach for decision-making during the early product design phase. Compared with other approaches or tools for the multi-objective conceptual design, this newly proposed approach has some unique advantages.
Table 9 The optimal solution selected based on three different TOPSIS method

\begin{tabular}{lllll}
\hline Solution & $\begin{array}{l}\text { Assembly } \\
\text { attribute }\end{array}$ & $\begin{array}{l}\text { Manufacturing } \\
\text { attribute }\end{array}$ & Cost attribute & Remarks \\
\hline Solution A & 63 & 59 & 38 & Expert weighting \\
Solution B & 45 & 50 & 21 & Information entropy weight \\
Solution C & 50 & 52 & 24 & The combined weight \\
\hline
\end{tabular}

Table 10 The module options of the optimal solution C

\begin{tabular}{llllll}
\hline Module & Impeller blade bending form & Blade type & Blade shape & Impeller structure & Impeller technology \\
\hline Option & Back bending form & Straight & Perforated & Semi-enclosed & Welding \\
Module & Casing & Coupling & Diaphragm & Shaft seal & bearing \\
Option & Vertical section & Flange & Bladeless & Carbon segmented & Step-land \\
\hline
\end{tabular}


1. With this approach, the feasible design solutions is generated from a complete solution space. According to morphological matrix, all the potential solutions are obtained in the form of module option.

2. With this approach, the multi-objective conceptual design process is divided into two stages: multiobjective optimization and selection. A large number of dominant solutions can be eliminated quickly through the optimization stage, which greatly reduces the screening workload of the optimal solution.

3. This approach can partly complete the evaluating works about the module properties and the final conceptual design scheme. The functional module properties are described quantitatively by corresponding attributes and scores. Therefore, the final conceptual scheme can be evaluated according to synthesis these module properties.

4. This approach has more flexibilities for the designers to emphasize their opinions and understanding about the product design task. In practical engineering practice, it is also necessary to prepare some appropriate standby schemes. At this time, we can obtain the corresponding standby scheme by adjusting the relevant parameters of MOPSO and TOPSIS.

\section{Concluding remarks}

The major goal of this work is to present a way for optimization and selecting the optimal multi-objective solution for comprehensively considering different product aspects (assembly, manufacturing and cost). In conceptual design stage, the multi-objective analysis of product assembly, manufacturing and cost supports design to obtain a tradeoff design solution. The case analysis (centrifugal compressor) presented in this article can effectively prove the feasibility and effectiveness of the proposed method.

Although this multi-objective analysis method can achieve the optimization and selection of the optimal conceptual solutions, there are still many places which can be furthermore improved. For example, the presented method can only generate conceptual scheme in the form of functional module option determination. However, it is difficult to deal with the non-option selection problem, such as the best value of component size. Additionally, the mapping between functional basis and modules is defined as one-to-one mapping in this article, it can be more suitable for practice conceptual design only if more complex and realistic mapping can be taken into consideration. All these problems will be considered in future work.

Acknowledgements This research is supported by the Funds for the National Natural Science Foundation of China (Grant No. 72072072), the Natural Science Foundation of Guangdong Province of China (Grant No. 2019A1515010045), the Outstanding Innovative Talents Cultivation Funded Programs for Doctoral Students of Jinan University (2021CXB027) and 2018 Guangzhou Leading Innovation Team Project, China (Grant No. 20190910006).

Funding Funding was provided by The Outstanding Innovative Talents Cultivation Funded Programs for Doctoral Students of Jinan University (2021CXB027), the Natural Science Foundation of Guangdong Province of China (Grant Nos. 2019A1515010045). The National Natural Science Foundation of China (72072072) and Guangzhou Research Collaborative Innovation Projects (20190910006).

\section{Declarations}

Conflicts of interest The authors declare no conflict of interest.

Ethical approval This article does not involve the content of violating ethics and morality.

Consent to participate and consent to publish All authors have read and agreed to participate and publish the manuscript.

Data availability The data used to support the finding of this article mainly included within this paper.

Open Access This article is licensed under a Creative Commons Attribution 4.0 International License, which permits use, sharing, adaptation, distribution and reproduction in any medium or format, as long as you give appropriate credit to the original author(s) and the source, provide a link to the Creative Commons licence, and indicate if changes were made. The images or other third party material in this article are included in the article's Creative Commons licence, unless indicated otherwise in a credit line to the material. If material is not included in the article's Creative Commons licence and your intended use is not permitted by statutory regulation or exceeds the permitted use, you will need to obtain permission directly from the copyright holder. To view a copy of this licence, visit http://creativecommons. org/licenses/by/4.0/.

\section{References}

1. Sassanelli C, Urbinati A et al (2020) Addressing circular economy through design for $\mathrm{X}$ approaches: a systematic literature review. Comput Ind. https://doi.org/10.1016/j.compind.2020.103245

2. Yadav DP, Patel DN, Morkos BW (2018) Development of product recyclability index utilizing design for assembly and disassembly principles. J Manuf Eng Transa Same. https://doi.org/10.1115/1. 4038515

3. Stone RB, McAdams DA (2004) A product architecture-based conceptual DFA technique. Des Stud 25:301-325

4. Favi C, Germani M (2012) A method to optimize assemblability of industrial product in early design phase: from product architecture to assembly sequence. Int J Interact Des Manuf 6(3):155-169

5. Pahl G, Beitz W (2013) Engineering design: a systematic approach. Springer, New York

6. AlGeddawy T, Samy SN, EIMaraghy H (2017) Best design granularity to balance assembly complexity and product modularity. J Eng Des 28:1-24 
7. Pahl G, Beitz W, Feldhusen J, Grote KH (2007) Engineering design: a systematic approach, 3rd edn. Springer, Berlin

8. Estorilio C, Simião MC (2006) Cost reduction of a diesel engine using the DFMA method. Product Manag Dev 4:95-103

9. Das SK, Datla V, Samir G (2000) DFQM-an approach for improving the quality of assembled products. Int J Product Res 38(2):57-477

10. Todić V, Lukić D, Milošević M, Jovičić G, Vukman J (2012) Manufacturability of product design regarding suitability for manufacturing and assembly (DfMA). J Prod Eng 16(1):47-50

11. Hoque ASM, Halder PK, Parvez MS (2013) Szecsi T (2013) Integrated manufacturing features and design-for-manufacture guidelines for reducing product cost under CAD/CAM environment. Comput Ind Eng 66:988-1003

12. Ma J, Kremer GEO (2016) A systematic literature review of modular product design (MPD) from the perspective of sustainability. Int J Adv Manuf Technol 86:1509-1539. https://doi.org/10.1007/ s00170-015-8290-9

13. Kumar PS, Behera H, Kumari KA, Nayak J, Naik B (2020) Advancement from neural networks to deep learning in software effort estimation: perspective of two decades. Comput Sci Rev 38:100288
14. Relich M (2016) Portfolio selection of new product projects: a product reliability perspective Ekspolatacja i Niezawodn. Maint Reliab 18:613-620

15. Kłosowski G, Gola A (2016) Risk-based estimation of manufacturing order costs with artificial intelligence. In: Proceedings of the 2016 federated conference on computer science and information systems, Gdansk, Poland, 11-14. IEEE: Gdansk, Poland, pp. 729-732 (2016).

16. Rajab S, Sharma V (2017) A review on the applications of neurofuzzy systems in business. Artif Intell Rev 49:481-510

17. Kim $\mathrm{N}$ et al (2019) Improving surface roughness of additively manufactured parts using a photopolymerization model and multi-objective particle swarm optimization. Appl Sci 9(1):151

18. Yue Z (2011) An extended TOPSIS for determining weights of decision makers with interval numbers. Knowl Based Syst 24:146-153

Publisher's Note Springer Nature remains neutral with regard to jurisdictional claims in published maps and institutional affiliations. 